\title{
Mitochondrial content reflects oocyte variability and fertilization outcome
}

\author{
Teresa Almeida Santos, Ph.D., ${ }^{\mathrm{a}}$ Shahy El Shourbagy, M.D., ${ }^{\mathrm{b}}$ and Justin C. St. John, Ph.D. ${ }^{\mathrm{b}}$ \\ ${ }^{a}$ Serviço de Genética Médica do Departamento de Medicina Materno-fetal, Genética e Reprodução Humana dos Hospitais da \\ Universidade de Coimbra and Faculdade de Medicina da Universidade de Coimbra, Coimbra, Portugal; and ${ }^{\mathrm{b}}$ Mitochondrial and \\ Reproductive Genetics Group, Medical School, University of Birmingham, Birmingham, United Kingdom
}

\begin{abstract}
Objective: To determine the content of mitochondrial DNA (mtDNA) in oocytes from a range of patients with fertilization success and failure.

Design: Analysis of mtDNA content in fertilized and unfertilized oocytes and embryos by real-time polymerase chain reaction (PCR).

Setting: University hospital infertility and research center.

Patient(s): Fifty-four women seeking treatment for infertility.

Intervention(s): None.

Main Outcome Measure(s): A total of 142 fertilized and unfertilized oocytes were classified into three main groups. Group I consisted of 35 fertilized oocytes from 21 patients; group II, 65 unfertilized oocytes from 36 patients; and group III, 42 degenerate oocytes from 23 patients. Mitochondrial DNA content was determined by SYBR Green real-time PCR-based assay.

Result(s): The mean mtDNA copy number for the fertilized oocytes was 250,454, whereas for the unfertilized group it was 163,698 . There were significant differences for mtDNA copy number between the male factor and female factor infertility unfertilized oocytes and between the unexplained infertility and female factor infertility groups. The mean copy number for the degenerate oocyte group was 44,629 , which was significantly different from the other subdivisions in this group.

Conclusion(s): Mitochondrial DNA content is critical to fertilization outcome and serves as an important marker of oocyte quality, explaining some cases of fertilization failure. (Fertil Steril ${ }^{\circledR} 2006 ; 85: 584-91$. C2006 by American Society for Reproductive Medicine.)
\end{abstract}

Key Words: Mitochondria, mitochondrial DNA, oocyte, embryo, fertilization, human

Many factors affect the fertilizability of an oocyte. In addition to male and female infertility, there is considerable unexplained or idiopathic infertility. Some of these cases might be explained by the failure of an oocyte to reach an appropriate level of maturity by the time it reaches metaphase II. For example, Eppig et al. (1) have proposed a cytoplasmic factor, mitosis-promoting factor, as a key regulating factor, whereas others have proposed protein kinase $\mathrm{C}$ (2) and mitogen-activating protein kinase activities (3). After hyperstimulation protocols and before fertilization programs, oocytes are assessed according to morphological criteria for suitability, including cytoplasmic arrangement (4).

Mitochondria are key players in cytoplasmic activity, providing, among other biochemical factors, adenosine triphosphate. Before the process of fertilization, mitochondria are dispersed throughout the cytoplasm and are dormant (5). After fertilization, however, each stage of cleavage necessi-

Received January 6, 2005; revised and accepted August 25, 2005.

Supported by the Egyptian Cultural and Educational Embassy Training Fellowship.

T.A.S. and S.E.L. contributed equally to this study.

Reprint requests: Justin C. St. John, Ph.D., University of Birmingham, Medical School, Mitochondrial and Reproductive Genetics Group, Birmingham B15 2TJ, United Kingdom (FAX: 44-121-414-6919; E-mail: j.stjohn.1@bham.ac.uk). tates that these highly dynamic organelles cluster around the nucleus to fuel cell division (6-8). After division, they disperse through the cytoplasm until the next round of cleavage. Consequently, these organelles are vital to subsequent embryonic development.

Intrinsically linked to mitochondrial function is mitochondrial DNA (mtDNA). This 16.6-kb double-stranded circular genome encodes 13 of the proteins of the electron transfer chain associated with the process of oxidative phosphorylation. Unlike somatic cells, each oocyte mitochondrion has only a single copy of this genome $(9,10)$, which suggests that it is representative of mitochondrial number. Indeed, throughout oogenesis and oocyte maturation, oocyte copy number is expanded from only 10 mitochondria at the primordial follicle stage to significantly larger numbers in mature oocytes (11). However, the probable large differences observed are due to inter-oocyte variability, highlighted by two studies reporting mean mtDNA copy numbers of 138,000 (12) and 314,000 (13) in unfertilized oocytes. A more recent study demonstrated that the average copy number from 113 oocytes that failed to fertilize ranged from 20,000 to 598,000 , with a mean of 193,000 copies (14).

This considerable exponential clonal expansion of mitochondria seems to discontinue once the oocyte has reached 
metaphase II and oocyte expansion and development have arrested (11). It is further discontinued in the fertilized oocyte, which tends not to increase copy number until after implantation $(10,15)$, though a recent finding suggests an early but brief window of mtDNA postfertilization replication (16). Consequently, each round of cleavage will result in each newly formed blastomere possessing fewer copies of the genome and hence mitochondria. This would suggest that a critical number of mitochondria are required for postfertilization events, especially given that the inability to trigger transcription and replication of mtDNA after implantation compromises fetal development, resulting in offspring harboring mtDNA-mediated cardiomyopathy or failing to survive in utero (15). Consequently, those oocytes possessing low mtDNA copy number are likely to be compromised before or after fertilization.

Here we report an investigation of whether mtDNA copy number influences fertilization outcome. In this instance, we analyzed the number of mitochondria present in oocytes that failed to fertilize, in oocytes that successfully fertilized, and in oocytes that degenerated either before or after fertilization. We have incorporated oocytes resulting from male and female factor infertility and from idiopathic infertility.

\section{MATERIALS AND METHODS Biological Material}

The unfertilized human oocytes and nontransferable embryos for mtDNA quantification were used from consenting patients undergoing fertility treatments involving both IVF and intracytoplasmic sperm injection (ICSI), according to appropriate institutional review board guidelines of the University Hospitals of Coimbra specific to the assisted reproductive technology program. The use of chaotic, highly fragmented embryos and abnormally fertilized oocytes was considered appropriate because these were not suitable for treatment purposes. None of the investigators had any conflict of interest.

\section{Ovarian Stimulation Protocol}

Each patient underwent the long down-regulation protocol. The GnRH agonist buserelin $(0.6 \mathrm{mg} /$ day SC) was started in the midluteal phase of the previous cycle (day 21), as determined by serum $E_{2}$ and $P$ concentrations for 12-14 days until pituitary ovarian down-regulation was reached (i.e., $\mathrm{E}_{2}$ concentration $<50 \mathrm{pg} / \mathrm{mL}$ ). Follicular growth was stimulated by FSH (Metrodin HP; Serono, Bari, Italy) or recombinant FSH (Gonal-F [Serono] or Puregon [Organon, Oss, The Netherlands]) (150-300 IU/day IM). This commenced after adequate down-regulation and continued by daily injections according to individual endocrine and ovarian ultrasonic response until at least one 18 -mm-diameter follicle was observed. Ovulation was induced with hCG (Pregnyl, Organon; 5,000 IU IM). Oocyte retrieval followed 34-36 hours later by ultrasound-guided transvaginal probe.

\section{IVF and ICSI Protocols}

Sperm for IVF and ICSI were prepared from ejaculated semen by passage through colloidal silica gradients (three layers of $40 \%, 70 \%$, and $90 \%$, or just one layer of $40 \%$ in cases of severe oligoasthenoteratospermia). Seminal fluid was removed by centrifugation for 15 minutes at $1,800 \times g$ and removal of the supernatant. After the addition of $5 \mathrm{~mL}$ of Earle's medium (Gibco BRL, Paisley, Scotland) and further centrifugation for 10 minutes at $1,800 \times g$, the supernatant was discarded, and sperm cells were allowed to migrate at $37^{\circ} \mathrm{C}$ for $10-60$ minutes in IVF medium. Before the ICSI procedure, the sperm suspension was diluted in a $10 \%$ polyvinylpyrrolidone solution (Sigma Chemical, St. Louis, MO).

Cumulus cells were removed by incubation in $25 \mathrm{IU}$ hyaluronidase (Choay, Sanofi Synthelabo, Paris, France) and intermittent pipetting. Oocytes were placed in 5- $\mu \mathrm{L}$ drops of IVF media with one drop of the sperm suspension and one drop of polyvinylpyrrolidone. Before oocyte injection, each motile sperm was immobilized by squeezing the tail between the bottom of the dish and the microinjection pipette. Microinjection was performed in IVF medium (Medicult, Jyllinge, Denmark) under mineral oil (Sigma Chemical) with holding and microinjection pipettes (Laboratoire CCD, Paris, France).

Insemination by IVF of the cumulus-intact oocytes was performed with a mean of 100,000 selected sperm cells. Injected and inseminated oocytes were cultured in IVF medium at $37^{\circ} \mathrm{C}$ in $5 \% \mathrm{CO}_{2}$, and fertilization was assessed after 16-18 hours. Cleavage was evaluated after a further 24 hours of in vitro culture. When extrusion of the second polar body and pronuclear formation were not observed at 48 hours, oocytes were considered unfertilized.

\section{Classification of the Oocyte Samples}

In all, 142 intact fertilized and unfertilized oocytes, zygotes, and embryos were analyzed. "Intact" was defined as the entire oocyte, zygote, or embryo. These were divided into three main groups: group I, fertilized oocytes $(n=35$ from 21 patients; mean age [range], 32 [24-38] years); group II, unfertilized oocytes $(\mathrm{n}=65$ from 36 patients, aged 32 [24-40] years); and group III, degenerate oocytes $(n=42$ from 23 patients, aged 31 [24-37] years).

In group I, fertilized oocytes were subdivided into three subgroups: group IA comprised 15 arrested two-pronuclei (2PN) zygotes collected from 7 patients; group IB, which included abnormally fertilized (3PN) zygotes or embryos, comprised $103 \mathrm{PN}$ zygotes, $14 \mathrm{PN}$, and 3 3PN six-cell embryos collected from 10 patients; group IC comprised 6 normally fertilized embryos (with abundant fragmentation) collected from 4 patients. Group I was further divided according to the cause of infertility, based on either male or female factor infertility. The male factor infertility group (group $\mathrm{ID}, \mathrm{n}=17$ ) comprised 6 abnormally fertilized zygotes (5 3PN and $14 \mathrm{PN}$ ), 3 abnormally fertilized embryos (six-cell 3PN embryos from a 3PN zygote), 6 2PN arrested normally fertil- 
ized zygotes, and 2 normally fertilized embryos with abundant fragmentation. The female factor infertility group (IE, $\mathrm{n}=18$ ) comprised 5 3PN zygotes, 9 2PN arrests, and 4 normally fertilized embryos with fragmentation.

Group II (unfertilized) included both IVF- and ICSItreated oocytes that were deemed unfertilized when no pronuclei or second polar bodies were observed after 48 hours of culture after insemination or microinjection. Group IIA (male factor infertility) comprised 24 unfertilized oocytes obtained from 15 patients (aged 32 [24-40] years). Fertilization failure arose from severe sperm defects, such as those patients classified as having severe oligoasthenozoospermia. Oocytes were deemed to be of fertilizable quality and did not exhibit characteristics of cytoplasmic fragmentation or cytoplasmic maturation failure. Some of these oocytes were part of a larger cohort from which it was decided to inseminate half of the oocytes and microinject the other half.

Group IIB (unexplained infertility) comprised 12 unfertilized oocytes obtained from 6 patients diagnosed as idiopathic with unassociated female or male factor defects. Oocytes were mature and of fertilizable quality with no cytoplasmic defects. Group IIC (female factor infertility) comprised 29 unfertilized oocytes obtained from 15 patients. Female infertility was diagnosed as due to endometriosis, polycystic ovary, elevated LH level, or anovulation. All oocytes were inseminated with normal sperm.

In group III (degenerate oocytes), 42 oocytes were obtained from 23 patients and were classified as degenerate either before or after IVF or ICSI procedures. They characteristically exhibited darkened cytoplasms.

\section{DNA Extraction}

Each intact oocyte, zygote, or embryo was placed in individual 2.0-mL cryovials (Sarstedt International, Rio de Mouro, Portugal) and stored at $-20^{\circ} \mathrm{C}$ until DNA extraction. Oocytes, zygotes, or embryos were thawed, and $20 \mu \mathrm{L}$ of autoclaved sterile $\mathrm{H}_{2} \mathrm{O}$ was added. The samples were then subjected to freezethaw disruption, as previously described $(17,18)$.

\section{Real-Time Polymerase Chain Reaction Analysis}

To determine the original starting copy number for each oocyte or embryo, the samples were subjected to quantification by real-time polymerase chain reaction (PCR). An external standard of 158-bp PCR product was generated, as previously described (14). Standards were purified with a commercial kit (QIAquick Gel Extraction Kit Protocol [DNA]; Qiagen, London, United Kingdom) according to the manufacturer's protocol. They then underwent spectrophotometry (Ultrospec 2000; Pharmacia Biotech, Hitchin, United Kindsom), and 10-fold dilutions were prepared. As previously described, $1 \mathrm{ng}$ of $158 \mathrm{PCR}$ product comprises $5.8 \times 10^{9}$ molecules of double-stranded DNA (14).

The master mix for each real-time PCR contained the following (total volume of $18 \mu \mathrm{L}$ ): $1 \times$ PCR buffer (Bio- line, London, United Kingdom), $25 \mathrm{mmol} / \mathrm{L}$ deoxynucleoside triphosphate (Bioline), $50 \mathrm{mmol} / \mathrm{L} \mathrm{MgCl}_{2}$ (Bioline), $0.5 \mu \mathrm{mol} / \mathrm{L}$ of each primer (D41 [nucleotide 3254-3277]: cga aag gac aag aga aat aag g; D56 [nucleotide 3126-3147]: ctg taa agt $\mathrm{ttt}$ aag $\mathrm{tt}$ tat gcg), 0.25 U SYBR Green, and $2 \mathrm{U}$ BioTaq DNA polymerase, supplemented with sterile water. Two microliters of each total DNA sample was added to individual wells of a 96-well PCR plate (Abgene, Cambridge, United Kindom), to which $18 \mu \mathrm{L}$ of the master mix was added. The reactions were preformed with the iCycler iQ Real Time Detection System (Bio-Rad Laboratories, Hercules, CA). The reaction conditions were 1 cycle at $95^{\circ} \mathrm{C}$ for 3 minutes, followed by $94^{\circ} \mathrm{C}$ for 30 seconds, $58^{\circ} \mathrm{C}$ for 30 seconds, and $72^{\circ} \mathrm{C}$ for 30 seconds, for 50 cycles. All reactions were run in triplicate and repeated on three separate occasions. The data obtained were analyzed with Microsoft Excel (Microsoft, Redmond, WA).

The iCycler software generated a standard curve using five 10-fold serial dilutions for each set of PCR reactions for the sought-after mtDNA target, using the primer pair D41 and D56. The starting copy number of mtDNA for each sample was determined by comparing the fluorescence intensity of the standards with the intensity of the unknown samples and adjusting for the $10 \times$ dilution factor. Melt curves were analyzed for any incidence of nonspecific priming. All PCR reactions were run on DNA gels to confirm the absence of primer dimerization.

\section{Statistical Analysis}

Student's $t$-test and one-way analysis of variance were performed on mtDNA copy number after logarithmic transformation of the data. All analyses were performed with commercial software (Minitab 13.1; Minitab, State College, PA).

\section{RESULTS}

In all, we analyzed 142 DNA samples from 54 patients with varying causes of infertility. These DNA samples were isolated from individual intact oocytes that either fertilized or failed to fertilize or were degenerate before or after insemination. Each of the DNA samples was then subjected to real-time PCR analysis to determine the number of mtDNA genomes per oocyte and whether an association existed between the number of mitochondria per oocyte and fertilization outcome. Each DNA sample was quantified against known standards, generated as described in Materials and Methods. Figure 1 is an example of the standard curves used to determine the number of copies present in individual oocytes, whereas Figure 2 demonstrates primer specificity through melt curve analysis, indicating that PCR products were not artifacts resulting from, for example, primer dimerization. The absence of primer dimerization was confirmed by gel electrophoresis (data not shown).

Group I (fertilized oocytes) consists of 35 zygotes or embryos collected from 21 patients (mean age [range], 32 
Real time PCR. Amplification of standards used to determine the number of mitochondria in a series of fertilized, unfertilized, and degenerate oocytes. Reaction conditions are described in the "Materials and Methods." Legend: negative (b/ue); 1 in 10 dilution (pink); 1 in 100 dilution (yellow); 1 in 1,000 dilution (aqua); 1 in 10,000 dilution (purple); and 1 in 100,000 dilution (dark red).

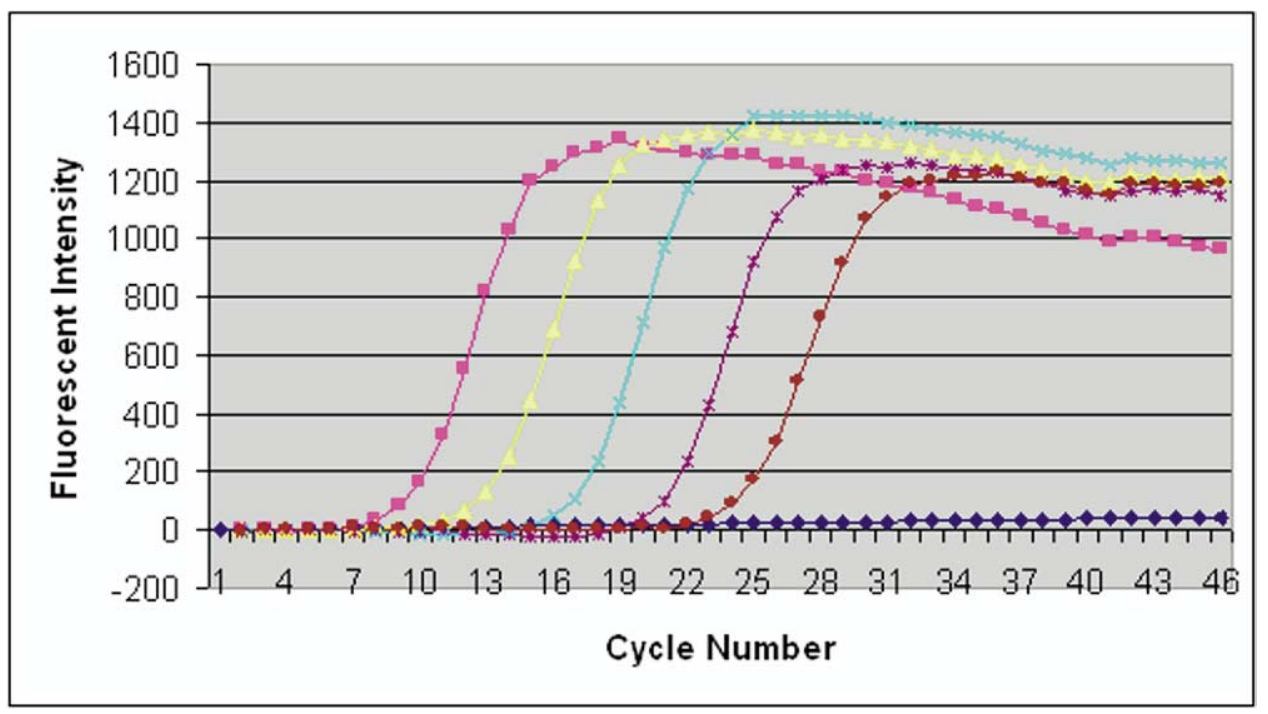

Santos. Mitochondria in oocytes and embryos. Fertil Steril 2006.

[24-38] years) with a mean mtDNA copy number of $250,454 \pm 29,730$ (Table 1). This group of fertilized oocytes was subdivided into three groups on the basis of their development in culture (Table 2). The 2PN arrested zygotes (group IA, $\mathrm{n}=15$ ) had a mean copy number of $165,260 \pm 126,853$, whereas the abnormally fertilized oocytes (group IB, $\mathrm{n}=14$ ) had a mean copy number of $306,000 \pm 174,513$. For the normally fertilized group (group IC, $\mathrm{n}=6$ ), the mean mtDNA copy number was $333,833 \pm 217,655$. This represented a significant difference for the mtDNA copy number between the three groups $(P<.02)$.

\section{FIGURE 2}

Melt curve analysis. Melt curves were constructed following each reaction to determine whether mispriming had occurred in any reactions by performing Sybr Green fluorescence reactions at temperatures between $55-93^{\circ} \mathrm{C}$. Any reactions perceived to contain mispriming were repeated to eliminate doubt.

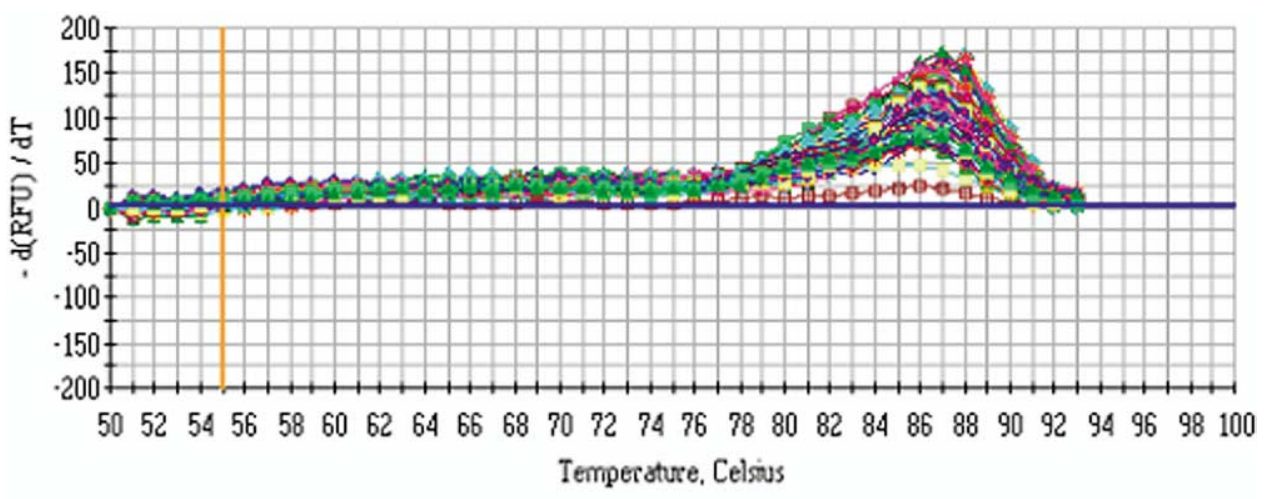

Mch Pcsk: justin hum 26-3.p.02 1050.opd

Santos. Mitochondria in oocytes and embryos. Fertil Steril 2006. 


\begin{tabular}{|c|c|c|c|c|}
\hline \multicolumn{5}{|c|}{ Mitochondrial DNA copy number for fertilized and unfertilized oocytes. } \\
\hline Group & No. of oocytes & Mean & Minimum & Maximum \\
\hline Fertilized & 35 & $250,454 \pm 29,730^{a}$ & 26,800 & 700,000 \\
\hline Unfertilized & 65 & $163,698 \pm 20,192$ & 14,100 & 700,000 \\
\hline \multicolumn{5}{|c|}{$\begin{array}{l}\text { Note: Values are means } \pm \text { SD. } \\
\text { a } P<.002 \text { vs. unfertilized group. }\end{array}$} \\
\hline
\end{tabular}

Group I was subdivided according to male or female factor as the cause of infertility. No significant difference was observed between these two groups (Table $3 ; P>.05$ ). The mean mtDNA copy number for the male factor group (group ID) was 247,694 $\pm 137,397$, compared with 253,061 $\pm 209,978$ for the female factor group (group IE). However, the pregnancy rate was higher in group ID (five pregnancies in 12 patients, $41.7 \%$ ) than in group IE (one pregnancy in 8 patients, $12.5 \%$ )

For the unfertilized oocyte group (group II, $\mathrm{n}=65$ from 36 patients, aged 32 [24-40] years) the mean mtDNA copy number was $163,698 \pm 20,192$. However, there was also considerable inter-oocyte variation (14,100 to 700,000 copies; Tables 1 and 4), even within individual cohorts. Compared with the mean of $250,454 \pm 29,730$ in fertilized oocytes for group I, the difference between the two groups was significant $(P<.002$; Table 1$)$. A comparison of group IIA (male factor infertility; 218,883 \pm 207,258 mean mtDNA copies) with group IIC (female factor infertility; $103,528 \pm 102,332$ mean mtDNA copies) revealed a significant difference $(P<.02)$. We also compared the mtDNA mean copy number for group IIB (unexplained infertility; $198,742 \pm 142,095$ copies) with that of group IIC (103,528 $\pm 102,332$ copies), which difference was also statistically significant $(P<.005$; Table 4). However, the difference in mean mtDNA copy number between groups IIA and IIB was not statistically significant ( $P>.05$; Table 4$)$.

The number of mtDNA genomes in the degenerate oocyte group (group III; $n=42 ; 44,629 \pm 40,729$ ) was statistically significantly different from the number in each of the other three infertility groups: IIA, $P<.001$ ); IIB, $P<.001$; IIC, $P<.001$ (Table 4). However, analysis of all the embryos revealed that there was no effect of age on mtDNA copy number. For those patients aged $<30$ years, $30-35$ years, and $>35$ years, mean mtDNA copy number was 254,133 $\pm 281,806,223,499 \pm 261,954$, and 149,658 $\pm 222,064$ copies, respectively $(P>.05)$. Surprisingly, there was no significant difference in mean mtDNA copy number between those patients aged $<33$ years $(229,885 \pm 280,790)$ and those aged $\geq 33$ years $(194,201 \pm 250,640)$. This is the defining age breakpoint for the acceptability of donors in many assisted reproductive technology programs and for the increase observed in the decline of embryo quality and implantation capacity.

\section{DISCUSSION}

It is evident from our data that a very strong association exists between the number of mitochondria, based on the number of mtDNA genomes present, and fertilization outcome. This seems to be independent of whether the oocyte shows normal patterns of division after fertilization (i.e., maintaining 2PN status) or fertilizes abnormally, resulting in 3PN status. We noted, however, that there is a significant difference in the mean mtDNA copy number for the types of fertilized oocytes analyzed, as shown in Table 2. Consequently, it would seem that normally fertilized oocytes are further distinct in their requirements for mitochondria.

\section{TABLE 2}

Mitochondrial DNA copy number in fertilized oocytes (arrested 2PN, abnormally fertilized oocytes, and normally fertilized oocytes).

\begin{tabular}{|lccrc|}
\hline Oocyte type & No. of oocytes & Mean & Minimum & Maximum \\
\hline Arrested 2PN & 15 & $165,260 \pm 126,853^{\mathrm{a}}$ & 26,800 & 477,000 \\
Abnormally fertilized 3PN & 14 & $306,000 \pm 174,513^{\mathrm{a}}$ & 100,000 & 700,000 \\
Normally fertilized & 6 & $333,833 \pm 217,655^{\mathrm{a}}$ & 130,000 & 700,000 \\
\hline Note: Values are means \pm SD. & & & & \\
a $P<.02$ vs. both other groups. & & & & \\
Santos. Mitochondria in oocytes and embryos. Fertil Steril 2006. & & & \\
\hline
\end{tabular}




\begin{tabular}{|c|c|c|c|c|}
\hline \multicolumn{5}{|c|}{ Mitochondrial DNA copy number in fertilized oocytes characterized by cause of infertility. } \\
\hline Cause of infertility & No. of oocytes & Mean & Minimum & Maximum \\
\hline Male factor & 17 & $247,694 \pm 137,397$ & 26,800 & 482,000 \\
\hline Female factor & 18 & $253,061 \pm 209,978$ & 34,500 & 700,000 \\
\hline \multicolumn{5}{|c|}{$\begin{array}{l}\text { Note: Values are means } \pm \text { SD. } \\
\text { There was no significant difference between groups. }\end{array}$} \\
\hline Santos. Mitochondria in oocyte & ryos. Fertil Steril 2006. & & & \\
\hline
\end{tabular}

The cause of infertility related to male or female factor infertility is again a key indicator of the importance of mitochondrial function during fertilization. As described by Reynier et al. (14), we confirm that a significant difference in mtDNA copy number exists between male and female factor infertility-sourced oocytes for those that failed to fertilize. These differences can be explained by severe sperm defects present in the male subfertility group accounting for fertilization failure and are certainly supported by the lack of a significant difference for mtDNA copy number observed in the fertilized oocytes for these groups. Equally important, it seems that oocyte degeneration is clearly marked by the lack of oocyte mitochondria (group III) and, when challenged either by maturation protocols after superovulation protocols or fertilization, these oocytes fail. We have previously argued that superovulation protocols will produce a considerable number of poor-quality oocytes for fertilization (19) because they supersede the natural selection mechanisms associated with oogenesis, such as atresia. Such events are designed to prevent these oocytes from being ovulated.

Our data are contrary to the recent finding by MayPanloup et al. (20), that the mean copy number of mitochondrial genomes was significantly lower in women with ovarian insufficiency compared with women with a normal ovarian profile. Although this emphasizes the need for a minimal number of mitochondrial genomes for normal ovarian development, it suggests that these findings are limited to patients with a particular ovarian profile. Our data suggest that this is consistent in ovaries from a variety of women, independent of whether their infertility relates to male or female factors. Indeed, atresia in oocytes could arise through either mtDNA depletion or through mtDNA deletions. Both embryos and oocytes possess variable numbers of mtDNA rearrangements $(21,22)$. This could account for the subsequent embryo development failure that led to the inception of cytoplasmic transfer, the supplementation of recipient oocytes with donor cytoplasm containing mitochondria (23).

Our data clearly indicate that there is a developmental failure restricting the clonal expansion of mtDNA molecules during oogenesis. To this extent, the expansion of mtDNA copy number has been described in cattle, with a 45 -fold increase from the primordial cell to preovulating oocytes (24). Clinically, this lack of mtDNA replenishment would resemble mtDNA depletion syndromes. These have been associated with two key nuclear-encoded mtDNA transcription and replication factors that regulate mtDNA copy number: mitochondrial transcription factor A (TFAM) (25) and polymerase $\gamma$ (26). Low levels of TFAM expression are associated with, for example infantile mitochondrial myopathy (27), familial mtDNA-associated liver disease (28),

\section{TABLE 4}

Mitochondrial DNA copy number in unfertilized oocytes, characterized by cause of infertility, and degenerate oocytes.

\begin{tabular}{|lcccc|}
\hline Cause of infertility & No. of oocytes & Mean & Minimum & Maximum \\
\hline Male factor & 24 & $218,883 \pm 207,258^{\mathrm{a}, \mathrm{b}}$ & 25,600 & 700,000 \\
Female factor & 29 & $103,528 \pm 102,332^{\mathrm{c}}$ & 14,100 & 440,000 \\
Unexplained & 12 & $198,742 \pm 142,095$ & 42,600 & 578,000 \\
Degenerate & 42 & $44,629 \pm 40,729^{\mathrm{d}}$ & 10,000 & 187,000 \\
\hline
\end{tabular}

Note: Values are means \pm SD.

${ }^{\text {a }} P>.05$ vs. unexplained group.

${ }^{\mathrm{b}} P<.02$ vs. female factor group.

${ }^{\mathrm{C}} P<.005$ vs. unexplained group.

${ }^{d} P<.001$ vs. other three groups.

Santos. Mitochondria in oocytes and embryos. Fertil Steril 2006. 
fatal childhood myopathy (29), skeletal muscle and mitochondrial encephalomyopathy disorders (30), and ocular myopathy, exercise intolerance, and muscle wasting (31).

The extent of TFAM control over mtDNA replication is decisive during mouse spermatogenesis, in which it is expressed up to the late spermatocyte/early spermatid stage. After this, it is then expressed as an alternative isoform, lacking the mitochondrial targeting sequence (32). Consequently, there is a 10 -fold reduction to approximately $70-80$ mtDNA copies per sperm (33). Indeed, the abnormal regulation of this strict developmental process is reflected in those subfertile men requiring assisted reproductive treatment, who consequently have significantly greater numbers of mtDNA copy number than fertile men (34). This reduction in mtDNA copy number has been suggested as a mechanism for restricting sperm mtDNA transmission after fertilization (32) and would thus be supported by the up-regulation observed in oocyte mtDNA copy number.

The regulation of TFAM expression is a precise phenomenon associated with early embryonic development. Mitochondrial DNA copy number remains unchanged until at least the blastocyst stage $(9,10,35)$, with each newly divided blastomere possessing fewer copies of the genome after each stage of embryonic division owing to there being no active mtDNA replication (see St. John et al. [36, 37] for review). Recently, the report of a small window of replication (16) might suggest that, for those oocytes with borderline copy number, a mechanism exists for partial rescue to bring the appropriate number of mitochondria above the fertilization threshold. However, blastomeres give rise to the inner cell mass that generate fetal tissue and are harvested to generate undifferentiated embryonic stem cell lines. This therefore necessitates that sufficient mtDNA be present for subsequent fetal development.

The precarious balance between the presence of the appropriate mtDNA copy number and subsequent fetal development is exemplified by outcomes associated with TFAM knockout mice. Homozygous TFAM knockout embryos exhibit severe mtDNA depletion and abolished oxidative phosphorylation. They proceed through implantation and gastrulation but die before embryonic day 10.5 (15). In contrast, heterozygous offspring present with mtDNA-depleted cardiomyopathy (38). Consequently, the low levels of mitochondria we have identified would be unlikely to provide sufficient mitochondria for fetal development because many of the inner cell mass cells would be depleted. Furthermore, insufficient numbers of mitochondria would be present to orchestrate the dynamic roles played by mitochondria during embryonic cellular division (6-8) and would likely trigger apoptosis due to inadequate adenosine triphosphate synthesis (39).

In many ways, loss of PolG, the polymerase unique to mtDNA replication, produces similar severe clinical consequences. This is perhaps best exemplified by its association with progressive external ophthalmoplegia (40). Furthermore, PolG activity can be severely inhibited by highly active antiretroviral therapy (HAART), used to reduce HIV load. Some of these regimens result in the generation of large-scale mtDNA deletions in mouse liver after azidothymidine administration (41). In sperm samples from HIV-positive men receiving HAART, we reported the accumulation of mtDNA deletions after 6 to 12 months of administration (42).

Similar occurrences have been reported in cell culture experiments on somatic cells (43) and can be mimicked through the administration of ethidium bromide to cell cultures (44). Dominant negative PolG expression (45) and targeted disruption of the exonuclease region of the gene (46) also induce an mtDNA depletion phenotype. The latter study also results in male infertility. PolG also possesses a series of CAG repeats, with the common allele consisting of 10 repeats. A recent study analyzing samples from cohorts of men throughout Europe has indicated an association between the absence of the common, 10-CAG-repeat allele and male subfertility (47).

Consequently, the clonal expansion of mtDNA through oogenesis (48) would suggest that for natural fertilization outcomes, a default mechanism exists that prevents those oocytes with insufficient numbers of mitochondria from successfully fertilizing. The maintenance of oocytes in metabolically supportive cultures might increase fertilization rates but could account for those women who have partial successful embryonic development but are then characterized by embryonic arrest. The introduction of donor oocyte cytoplasm has enhanced fertilization outcome for these women but raises several major moral and ethical issues $(19,49)$. These include the possibility of bimaternal inheritance $(50,51)$ and developmental abnormalities (52). This very much matches concerns related to chromosomal nondysjunction (53).

\section{REFERENCES}

1. Chesnel F, Eppig JJ. Synthesis and accumulation of p34cdc2 and cyclin $\mathrm{B}$ in mouse oocytes during acquisition of competence to resume meiosis. Mol Reprod Dev 1995;40:503-8.

2. Viveiros MM, Hirao Y, Eppig JJ. Evidence that protein kinase C (PKC) participates in the meiosis I to meiosis II transition in mouse oocytes. Dev Biol 2001;235:330-42.

3. Fan HY, Li MY, Tong C, Chen DY, Xia GL, Song XF, et al. Inhibitory effects of cAMP and protein kinase $\mathrm{C}$ on meiotic maturation and MAP kinase phosphorylation in porcine oocytes. Mol Reprod Dev 2002;63: $480-7$.

4. Veeck L. Atlas of the human oocyte and early conceptus. 1st ed. Philadelphia: Williams \& Wilkins, 1986.

5. Allen JF. Separate sexes and the mitochondrial theory of ageing. J Theor Biol 1996;180:135-40.

6. Squirrell JM, Lane M, Bavister BD. Altering intracellular $\mathrm{pH}$ disrupts development and cellular organization in preimplantation hamster embryos. Biol Reprod 2001;64:1845-54.

7. Bavister BD, Squirrell JM. Mitochondrial distribution and function in oocytes and early embryos. Hum Reprod 2000;15 Suppl 2:189-98.

8. Sun QY, Wu GM, Lai L, Park KW, Cabot R, Cheong HT, et al. Translocation of active mitochondria during pig oocyte maturation, fertilization and early embryo development in vitro. Reprod 2001;122: 155-63.

9. Piko L, Matsumoto L. Number of mitochondria and some properties of mitochondrial DNA in the mouse egg. Dev Biol 1976;49:1-10. 
10. Piko L, Taylor KD. Amounts of mitochondrial DNA and abundance of some mitochondrial gene transcripts in early mouse embryos. Dev Biol 1987; 123:364-74.

11. Jansen RP, de Boer K. The bottleneck: mitochondrial imperatives in oogenesis and ovarian follicular fate. Mol Cell Endocrinol 1998;145:81-8.

12. Chen X, Prosser R, Simonetti S, Sadlock J, Jagiello G, Schon EA. Rearranged mitochondrial genomes are present in human oocytes. Am J Hum Genet 1995;57:239-47.

13. Steuerwald N, Barrit JA, Adler R, Malter H, Schimmel T, Cohen J, et al. Quantification of mtDNA in single oocytes, polar bodies and subcellular components by real-time rapid cycle fluorescence monitored PCR. Zygote 2000;9:209-15.

14. Reynier P, May-Panloup P, Chretien MF, Morgan CJ, Jean M, Savagner F, et al. Mitochondrial DNA content affects the fertilizability of human oocytes. Mol Hum Reprod 2001;7:425-9.

15. Larsson NG, Wang J, Wilhelmsson H, Oldfors A, Rustin P, Lewandoski $\mathrm{M}$, et al. Mitochondrial transcription factor A is necessary for mtDNA maintenance and embryogenesis in mice. Nat Genet 1998;18:231-6.

16. McConnell JM, Petrie L. Mitochondrial DNA turnover occurs during preimplantation development and can be modulated by environmental factors. Reprod Biomed Online 2004;9:418-24.

17. Findlay I, Lilford R. Sources and detection of contamination in preimplantation diagnosis. In: Proceedings of XIII Annual Scientific Meeting of Fertility Society of Australia, Brisbane, Australia, October 1994:101.

18. Findlay I, Atkinson G, Chambers M, Quirke P, Campbell J, Rutherford A. Rapid genetic diagnosis at 7-9 weeks gestation: diagnosis of sex, single gene defects and DNA fingerprint from coelomic samples. Hum Reprod 1996;11:2548-53.

19. St. John JC. Ooplasm donation in humans: the need to investigate the transmission of mitochondrial DNA following cytoplasmic transfer. Hum Reprod 2002;17:1954-8.

20. May-Panloup P, Chrétien MF, Jacques C, Vasseur C, Malthièry Y, Reynier P. Low oocyte mitochondrial DNA content in ovarian insufficiency. Hum Reprod 2005;3:593-7.

21. Barritt JA, Brenner CA, Cohen J, Matt DW. Mitochondrial DNA rearrangements in human oocytes and embryos. Mol Hum Reprod 1999;5:27-33.

22. Brenner CA, Wolny YM, Barritt JA, Matt, DW, Munne S, Cohen J. Mitochondrial DNA deletion in human oocytes and embryos. Mol Hum Reprod 1998;4:887-92.

23. Cohen J, Scott R, Schimmel T, Levron J, Willadsen S. Birth of infant after transfer of anucleate donor oocyte cytoplasm into recipient eggs. Lancet 1997;350:186-7.

24. Smith LC, Alcivar AA. Cytoplasmic inheritance and its effects on development and performance. J Reprod Fertil Suppl 1993;48:31-43.

25. Fisher RP, Clayton DA. Purification and characterization of human mitochondrial transcription factor 1. Mol Cell Biol 1988;8:3496-509.

26. Ropp PA, Copeland WC. Cloning and characterization of the human mitochondrial DNA polymerase, DNA polymerase gamma. Genomics 1996;36:449-58.

27. Poulton J, Morten K, Freeman-Emmerson C, Potter C, Sewry C, Dubowitz V, et al. Deficiency of the human mitochondrial transcription factor h-mtTFA in infantile mitochondrial myopathy is associated with mtDNA depletion. Hum Mol Genet 1994;3:1763-9.

28. Spelbrink JN, Van Galen MJ, Zwart R, Bakker HD, Rovio A, Jacobs HT, Van den Bogert C. Familial mitochondrial DNA depletion in liver: haplotype analysis of candidate genes. Hum Genet 1998;102:327-31.

29. Larsson NG, Oldfors A, Holme E, Clayton DA. Low levels of mitochondrial transcription factor A in mitochondrial DNA depletion. Biochem Biophys Res Commun 1994;200:1374-81.

30. Siciliano G, Mancuso M, Pasquali L, Manca ML, Tessa A, Ludice A. Abnormal levels of human mitochondrial transcription factor $\mathrm{A}$ in skeletal muscle in mitochondrial encephalomyopathies. Neurol Sci 2000;21(5 Suppl):S985-7.

31. Tessa A, Manca ML, Mancuso M, Renna MR, Murri L, Martini B, et al. Abnormal H-Tfam in a patient harboring a single mtDNA deletion. Funct Neurol 2000;15:211-4.
32. Larsson NG, Garman JD, Oldfors A, Barsh GS, Clayton DA. A single mouse gene encodes the mitochondrial transcription factor $\mathrm{A}$ and a testis-specific nuclear HMG-box protein. Nat Genet 1996;13:296-302.

33. Hecht NB, Liem H. Mitochondrial DNA is synthesized during meiosis and spermiogenesis in the mouse. Exp Cell Res 1984;154:293-8.

34. May-Panloup P, Chretien MF, Savagner F, Vasseur C, Jean M, Malthiery $\mathrm{Y}$, et al. Increased sperm mitochondrial DNA content in male infertility. Hum Reprod 2003;18:550-6.

35. Ebert KM, Liem H, Hecht NB. Mitochondrial DNA in the mouse preimplantation embryo. J Reprod Fertil 1988;82:145-9.

36. St. John JC, Lloyd R, El Shourbagy S. The potential risks of abnormal transmission of mtDNA through assisted reproductive technologies. Reprod Biomed Online 2004;8:34-44.

37. St. John JC, Lloyd RE, Bowles EJ, Thomas EC, El Shourbagy S. The consequences of nuclear transfer for mammalian foetal development and offspring survival. A mitochondrial DNA perspective. Reproduction 2004;127:631-41.

38. Li H, Wang J, Wilhelmsson H, Hansson A, Thoren P, Duffy J, et al. Genetic modification of survival in tissue-specific knockout mice with mitochondrial cardiomyopathy. Proc Natl Acad Sci U S A 2000;97: 3467-72.

39. Van Blerkom J, Davis P, Alexander S. A microscopic and biochemical study of fragmentation phenotypes in stage-appropriate human embryos. Hum Reprod 2001;16:719-29.

40. Van Goethem G, Dermaut B, Lofgren A, Martin JJ, Van Broeckhoven C. Mutation of POLG is associated with progressive external ophthalmoplegia characterized by mtDNA deletions. Nat Genet 2001;28:211-2.

41. Hayakawa M, Ogawa T, Sugiyama S, Tanaka M, Ozawa T. Massive conversion of guanosine to 8-hydroxy-guanosine in mouse liver mitochondrial DNA by administration of azidothymidine. Biochem Biophys Res Comm 1991;176:87-93.

42. White DJ, Mital D, Taylor S, St. John JC. Sperm mitochondrial DNA deletions as a consequence of long term highly active antiretroviral therapy. AIDS 2001;15:1061-2.

43. Walker UA, Venhoff N, Koch EC, Olschewski M, Schneider J, Setzer B. Uridine abrogates mitochondrial toxicity related to nucleoside analogue reverse transcriptase inhibitors in HepG2 cells. Antiviral Ther 2003;8:463-70.

44. King MP, Attardi G. Isolation of human cells lacking mitochondrial DNA. Methods Enzymol 1996;264:304-13.

45. Jazayeri M, Andreyev A, Will Y, Ward M, Anderson CM, Clevenger W. Inducible expression of a dominant negative DNA polymerasegamma depletes mitochondrial DNA and produces a rho0 phenotype. J Biol Chem 2003;278:9823-30.

46. Trifunovic A, Wredenberg A, Falkenberg M, Spelbrink JN, Rovio AT, Bruder CC, et al. Premature ageing in mice expressing defective mitochondrial DNA polymerase. Nature 2004;429:417-23.

47. Rovio AT, Marchington DR, Donat S, Schuppe HC, Abel J, Fritsche E, et al. Mutations at the mitochondrial DNA polymerase (POLG) locus associated with male infertility. Nat Genet 2001;29:261-2.

48. Marchington DR, Hartshorne GM, Barlow D, Poulton J. Homopolymeric tract heteroplasmy in mtDNA from tissues and single oocytes: support for a genetic bottleneck. Am J Hum Genet 1997;60:408-16.

49. St. John JC, Barratt CLR. Use of anucleate donor oocyte cytoplasm in recipient egg. Lancet 1997;350:961-2.

50. Barritt JA, Brenner CA, Malter HE, Cohen J. Mitochondria in human offspring derived from ooplasmic transplantation. Hum Reprod 2001; 16:513-6.

51. Brenner CA, Barritt JA, Willadsen S, Cohen J. Mitochondrial DNA heteroplasmy after human ooplasmic transplantation. Fertil Steril 2000; 74:573-8.

52. Barritt J, Brenner C, Malter H, Cohen J. Rebuttal: interooplasmic transfers in humans Reprod Biomed Online 2001;3:47-8.

53. Schon EA, Kim SH, Ferreira JC, Magalhaes P, Grace M, Warburton D, et al. Chromosomal non-disjunction in human oocytes: is there a mitochondrial connection? Hum Reprod 2000;15 Suppl 2:160-72. 